ECCOMAS

Proceedia
COMPDYN 2021

$8^{\text {th }}$ ECCOMAS Thematic Conference on Computational Methods in Structural Dynamics and Earthquake Engineering M. Papadrakakis, M. Fragiadakis (eds.) Streamed from Athens, Greece, 28 - 30 June 2021

\title{
LOCAL SCALE DAMPING MODEL FOR REINFORCED CONCRETE ELEMENTS
}

\author{
Clotilde Chambreuil $^{1}$, Cédric Giry ${ }^{1}$, Frédéric Ragueneau ${ }^{1}$, and Pierre Léger $^{2}$ \\ ${ }^{1}$ Université Paris-Saclay, ENS Paris-Saclay, CNRS, LMT - Laboratoire de Mécanique et Technologie, \\ 91190, Gif-sur-Yvette, France. \\ e-mail: \{clotilde.chambreuil,cedric.giry,frederic.ragueneau\}@ens-paris-saclay.fr \\ 2 École Polytechnique de Montréal, Department of Civil, Geological and Mining Engineering, \\ C.P. 6079, succ. Centre-Ville, Montréal, Québec, Canada, H3C 3A7. \\ e-mail: pierre.leger@polymtl.ca
}

\begin{abstract}
Reinforced concrete $(R C)$ structures dissipate energy when subjected to seismic excitations. Modeling seismic energy dissipations on a rational basis still represents challenging issues. At local scale, dissipative constitutive RC models are developed to take into consideration different phenomena, such as cracks opening, friction, plasticity, and their couplings or rebar bond slip. Practically some of those dissipative mechanisms are modeled by equivalent Rayleigh viscous damping at the global structural level. However, Rayleigh damping lacks a physical basis leading sometimes to an uncontrolled evolution of energy dissipation when non linearities occur [1]. This paper provides the basis of an updated local damping model based on parameters modeling concrete dissipative phenomenon, to reduce the need for arbitrary equivalent viscous damping as much as possible and thus better model energy dissipations on a rational physical basis. Using shake table experimental results on prismatic RC beams [2], a numerical study is presented to assess the performance of sixteen different global damping formulations to represent physical energy dissipation mechanisms. Energy balance analyses are used to evaluate dissipative phenomena at the local concrete level and to demonstrate that friction is the most significant local dissipative phenomenon. In addition, an equivalent viscous damping identification method is developed to determine transient damping ratio evolution during dynamic computations as a function of damage parameters, like stiffness degradation. An exponential function is found suitable to take into consideration global viscous damping adjustment as a function of local damage occurring during dynamic nonlinear analyses.
\end{abstract}

Keywords: Damping, Reinforced Concrete, Dynamic, Nonlinear Model, Energy Balances.

ISSN:2623-3347 (C) 2021 The Authors. Published by Eccomas Proceedia.

Peer-review under responsibility of the organizing committee of COMPDYN 2021.

doi: $10.7712 / 120121.8790 .18761$ 


\section{INTRODUCTION}

Earthquake events in recent years lead to the need to evaluate structural vulnerability and to quantify related safety margins. Particularly, the combination of critical infrastructures presence on territories and more stringent seismic regulations represent a current challenge. Nuclear power plants are representing such critical structures. In the aim of increasing their lifespan, analyses are required to ensure their safety under current and evolving standards. For example, after a moderate earthquake excitation, the nuclear containment vessel still need to demonstrate airtightness. But, the difficulty with reinforced concrete structures is related to the lack of knowledge about concrete cracking damage and about the ability of the material to dissipate seismic energy.

To improve dynamic non-linear computations of complex structures under earthquake excitations, rational physical models need to be developed. To model dissipations during earthquake events, different scales are considered. First, at local scale, physical phenomena can be described through material constitutive laws. Then, at global scale, energy dissipations are also occurring because of the structure interaction with its environment. The impossibility to model all phenomena at local and structural scales leads to the use of equivalent viscous damping. Following Rayleigh's works [3], a large a mount of viscous damping matrix formulations have been developed in the literature. However, those formulations still lack a sound physical basis.

Based on a shake table experimental campaign performed by Heitz [2] to characterize seismic energy dissipation in reinforced concrete beams, a numerical study is presented herein. It aims at evaluating the performances of existing local as well as global (Rayleigh) damping formulations in representing the experimental data. The focus is put in dissipative evolutions at local and global scales. An identification method is also developed to study evolution of the equivalent viscous damping ratio during non-linear computations.

\section{REINFORCED CONCRETE BEAM UNDER EARTHQUAKE EXCITATION}

The experimental campaign performed by [2] at the French Alternative Energies and Atomic Energy Commission (CEA) Saclay represents a huge amount of data that can be used as a reference to validate numerical analyses. Experiments were performed with different material properties, structural configurations and excitation characteristics on reinforced concrete beam. The main objective was to evaluate seismic energy dissipations mechanisms.

\subsection{Beam model}

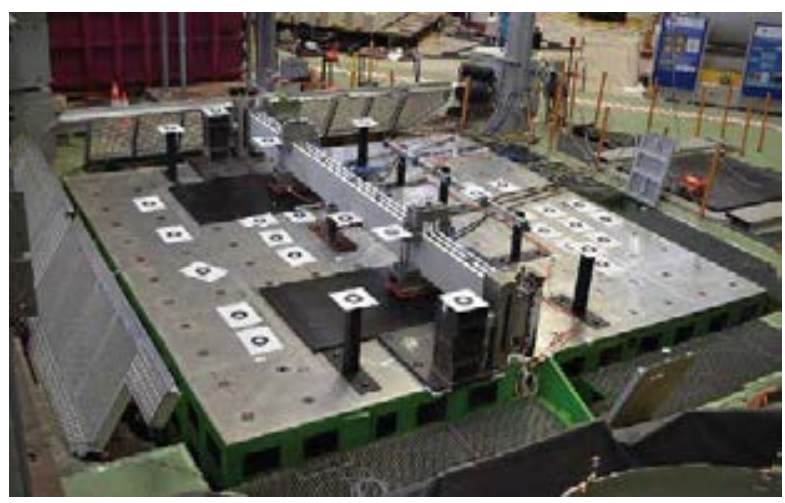

Figure 1: Dynamic experimental setup
Based on information about the experimental setup (fig. 1), a numerical model of the tested reinforced concrete beams has been developed using Cast $3 \mathrm{~m}$, a finite-element software. The main advantages of using a multifiber models are: (i) to avoid solid 3D models, limiting the number of degrees-of-freedom and so to save computational time, (ii) to consider 1D constitutive material models, which are easier to implement and unable to model local behaviors, and (iii) its usability for results interpretation $[4,5,6]$. Multi-fiber beam elements are based on Timoshenko beam the- 
ory in Cast3m.

A typical model geometry is presented in figure 2. Along the beam, sections are considered at each Gauss point of the beam elements (fig. 2a). Then, each section is decomposed in fiber elements. Figure $2 b$ shows the section model with concrete and steel elements. Those elements are $2 \mathrm{D}$ linear quadrangle elements.

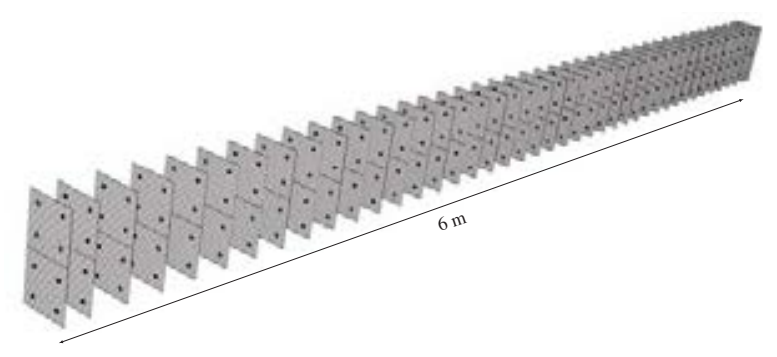

(a) Beam

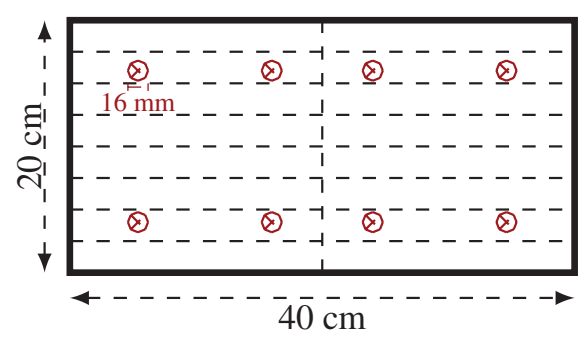

(b) Section

Figure 2: Beam geometry and discretization

To match experimental boundary conditions and beam undamaged properties, spring elastic supports are considered. Spring rotational and translational stiffnesses are calibrated from modal analyses performed on undamaged beam, considering a global symmetrical problem. The aim is to match the two first numerical eigen-frequencies with those deduced from experimental hammer shock tests. Finally, additional masses are added at the quarter and three-quarter beam span, and vertical displacements are blocked at those nodes. For quasi-static analyses, displacements are imposed at additional masses positions, and for dynamic computations, a force equivalent loading is derived from measured table accelerations.

\subsection{Material constitutive models}

Concrete model Two hysteretic models developed in Cast $3 \mathrm{~m}$ are compared in numerical studies. First a simple model, named BARFRA, was proposed by Crambuer [7]: the only dissipative phenomena considered is concrete cracking. The second model, named RICBET and developed by Richard [8] is more complex because it considers four dissipative phenomena: (i) cracking and (ii) friction in tension, (iii) plasticity in compression, as well as (iv) the unilateral effect dissipating energy upon crack closing. Thermodynamic potentials of both models are respectively expressed in equations 1 and 2. Computations of energy dissipations in section 3.1 of the paper will be deduced from those equations.

$$
\rho \psi=\frac{1}{2}\left(1-d_{+}\right)\left\langle\varepsilon_{i j}\right\rangle_{+} C_{i j k l}\left\langle\varepsilon_{k l}\right\rangle_{+}+H_{+}\left(z_{+}\right)+\frac{1}{2}\left(1-d_{-}\right)\left\langle\varepsilon_{i j}\right\rangle_{-} C_{i j k l}\left\langle\varepsilon_{k l}\right\rangle_{-}+H_{-}\left(z_{-}\right)
$$

with $\left\langle\varepsilon_{i j}\right\rangle_{+}$and $\left\langle\varepsilon_{i j}\right\rangle_{-}$respectively the positive and negative parts of the strain tensor, $C_{i j k l}$ the elastic tensor, $d_{+}$and $d_{-}$the damage index representing concrete cracking respectively for positive and negative strains and $H_{+}\left(z_{+}\right)$and $H_{-}\left(z_{-}\right)$the functions representing isotropic work 
hardening respectively for positive and negative strains.

$$
\begin{aligned}
\rho \Psi & =\frac{1}{2}\left\{(1-D)\left(\varepsilon_{i j}-\varepsilon_{i j}^{p}\right) C_{i j k l}\left(\varepsilon_{k l}-\varepsilon_{k l}^{p}\right)\right\}+\frac{1}{2}\left\{D\left(\varepsilon_{i j}-\eta \varepsilon_{i j}^{\pi}-\varepsilon_{i j}^{p}\right) C_{i j k l}\left(\varepsilon_{k l}-\eta \varepsilon_{i j}^{\pi}-\varepsilon_{k l}^{p}\right)\right\} \\
& +\frac{1}{2}\left\{\gamma \alpha_{i j} \alpha_{i j}\right\}+H(z)+G(p)
\end{aligned}
$$

with $D$ the damage index representing concrete tensile cracking, $\varepsilon_{i j}^{p}$ the plastic strain tensor, $\eta$ the variable associated to unilateral effect, $\varepsilon_{i j}^{\pi}$ the strain tensor associated to friction, $\gamma$ a parameter characterizing friction, $\alpha_{i j}$ the tensor of kinematic work hardening (friction phenomenon) and $H(z)$ and $G(p)$ the functions representing isotropic work hardening (damage and plasticity phenomena).

Both models are calibrated on the experimental data obtained from quasi-static tests. The advantage of using the most complex model is to better represent hysteresis cycles.

Steel model For steel reinforcements a classical elastoplastic model is used. Typical parameter values are considered. Indeed, experimental tests were developed to avoid reaching nonlinearities in steel reinforcements, to represent what happens to structures subjected to moderate earthquake accelerations.

\subsection{Dynamic problem}

General equation After calibrating the model with quasi-static tests, non-linear dynamic computations can be performed. Dynamic equilibrium equation for a multi-degree-of-freedom (MDOF) nonlinear damped system is:

$$
\mathbb{M} \cdot \ddot{\mathbf{U}}+\mathbb{C} \cdot \dot{\mathbf{U}}+\mathbf{f}_{i n t}(\mathbf{U})=-\mathbb{M} \cdot \underline{\Gamma} \cdot \ddot{\mathbf{U}}_{s}
$$

with $\mathbb{M}$ the mass matrix, $\mathbb{C}$ the damping matrix, $\ddot{U}, \dot{U}$ and $\mathbf{U}$ respectively the acceleration, velocity and displacement of beam nodes, $\mathbf{f}_{i n t}(\mathbf{U})$ the internal force characterizing the nonlinear constitutive model, $\ddot{\mathrm{U}}_{s}$ the seismic accelerations and $\underline{\Gamma}$ is the influence vector given the direction of seismic excitation. So, only the damping matrix $\mathbb{C}$ is unknown if $\mathbf{f}_{\text {int }}$ can be assessed with confidence.

Focus on damping matrix formulations The most frequently used formulation for the damping matrix derived from Rayleigh's work [3]. It consists in a linear combination of mass and initial stiffness matrices. Mathematically, this model leads to a decoupled problem using the undamped modal basis enabling its solving. However, numerous papers present the limits of this damping model, synthesized by Hall [1]. That is why, the literature presents different alternative for Rayleigh damping formulation that improve computations by minimizing some Rayleigh damping limitations. Particularly, we can find the use of tangent or secant stiffness matrix instead of initial ones [9]. This requires recomputing the damping matrix several time during analysis, increasing computational time. But it is a way to consider non-linear behavior in Rayleigh damping. On the other hand, it was demonstrated that the mass proportional part leads to unrealistic damping forces because higher modes are very lightly undamped. So, some papers propose to remove the mass matrix to keep only the stiffness proportional part given a linear correlation between the damping ratio of each mode and its frequency [10]. 
In addition to Rayleigh-type formulations, modal damping formulations are often used in the literature. In those cases, the $\mathbb{C}$ matrix is computed from modes determined from a modal analysis. For example, Wilson \& Penzien [11] proposed such a formulation, as well as Chopra $\&$ McKenna [12] based on the choice of a damping ratio for $N$ modes.

Herein, sixteen equivalent viscous damping formulations have been studied and compared in relation to each other, but also with different damping ratios, and with or without parameters updating. When parameters are updated during computation, modal analyses are performed at each time step and the new frequencies are considered to compute the damping matrix. Table 1 presents the classification and name of all studied Rayleigh type and modal damping formulations.

\begin{tabular}{|c|c|c|c|c|c|c|c|}
\hline & Formulation & Modes* & $\begin{array}{c}\text { Parameter } \\
\text { updating }\end{array}$ & & Formulation & Modes* & $\begin{array}{c}\text { Parameter } \\
\text { updating }\end{array}$ \\
\hline RD & $\begin{array}{l}\text { Classical Rayleigh } \\
\text { damping }\end{array}$ & 1 and 2 & $x$ & CRD & $\begin{array}{l}\text { Commit-stiffness } \\
\text { Rayleigh damping }\end{array}$ & 1 and 2 & $x$ \\
\hline RD_ACT & $\begin{array}{c}\text { Classical Rayleigh } \\
\text { damping }\end{array}$ & 1 and 2 & $\checkmark$ & CRD_ACT & $\begin{array}{l}\text { Commit-stiffness } \\
\text { Rayleigh damping }\end{array}$ & 1 and 2 & $\checkmark$ \\
\hline MPD & $\begin{array}{l}\text { Mass proportional } \\
\text { damping }\end{array}$ & 1 & $x$ & SRD & $\begin{array}{l}\text { Secant-stiffness } \\
\text { Rayleigh damping }\end{array}$ & 1 and 2 & $x$ \\
\hline MPD_ACT & $\begin{array}{l}\text { Mass proportional } \\
\text { damping }\end{array}$ & 1 & $\checkmark$ & MD & Modal damping & 1 to 6 & $x$ \\
\hline KPD & $\begin{array}{c}\text { Initial-stiffness } \\
\text { proportional damping }\end{array}$ & 1 & $x$ & MD_ACT & Modal damping & 1 to 6 & $\checkmark$ \\
\hline KPD_ACT & $\begin{array}{l}\text { Initial-stiffness } \\
\text { proportional damping }\end{array}$ & 1 & $\checkmark$ & WPD & Wilson-Penzien damping & 1 to 6 & $x$ \\
\hline CKPD & $\begin{array}{l}\text { Commit-stiffness } \\
\text { proportional damping }\end{array}$ & 1 & $x$ & CWPD & $\begin{array}{l}\text { Commit-stiffness } \\
\text { Wilson-Penzien damping }\end{array}$ & 1 and 2 & $x$ \\
\hline SKPD & $\begin{array}{l}\text { Secant-stiffness } \\
\text { proportional damping }\end{array}$ & 1 & $x$ & SWPD & $\begin{array}{l}\text { Secant-stiffness } \\
\text { Wilson-Penzien damping }\end{array}$ & 1 and 2 & $x$ \\
\hline
\end{tabular}

* Modes 1 and 2 represents $89.6 \%$ of the beam effective modal mass.

Table 1: Global damping matrices $(\mathbb{C})$ nomenclature

\section{THEORETICAL DEVELOPMENTS}

Using the developed multi-fiber model, the aim of this section is to focus on seismic energy dissipation-computations and transient damping evolution.

\subsection{Energy analyses}

Structural level Energy balance is deduced from the integration of dynamic equations (eq. 4) [13], corresponding to equation 5 , in absolute formalism:

$$
\begin{gathered}
\frac{1}{2}\left(\mathbb{M} \cdot \dot{\mathbf{U}}_{a}\right) \cdot \dot{\mathbf{U}}_{a}+\int(\mathbb{C} \cdot \dot{\mathbf{U}}) \cdot \dot{\mathbf{U}} \mathrm{d} t+\int \mathbf{f}_{i n t}(\mathbf{U}) \cdot \mathrm{d} \mathbf{U}=\int\left(\mathbb{M} \cdot \ddot{\mathbf{U}}_{a}\right) \cdot \mathrm{d}_{s} \\
E_{k, a}+E_{d}+E_{a}=E_{i, a}
\end{gathered}
$$

where $E_{k, a}, E_{d}, E_{a}$ and $E_{i, a}$ are respectively the absolute kinematic energy, the viscous damping energy, the absorbed energy (or internal energy) and the absolute injected energy. In the case of a linear behavior, the three left terms are easily computed respectively from mass, damping and stiffness matrices. However, when a nonlinear constitutive model is considered, the absorbed energy is decomposed between the strain energy (corresponding to the elastic part) and the hysteretic energy deduced from thermodynamical approach. The right energy balance term, the injected energy, corresponds to the energy induced by the seismic acceleration. 
Local level At material level, the hysteretic energy is now defined for both constitutive models in Table 2, with phenomenon and associated variables presented in Table 3 for BARFRA model and in Table 4 for RICBET model.

\begin{tabular}{|c|c|c|c|}
\hline \multirow{2}{*}{ Name } & \multirow{2}{*}{ Symbol } & \multicolumn{2}{|r|}{ Equation } \\
\hline & & BARFRA & RICBET \\
\hline Hysteretic energy & $E_{h}$ & $\int_{\mathscr{V}}(Y . \dot{d}) \mathrm{d} \mathscr{V}$ & $\int_{\mathscr{V}}\left(\sigma_{c} \cdot \dot{\varepsilon}_{c}^{p}+\sigma^{\pi} \cdot \dot{\varepsilon}^{\pi}+Y \cdot \dot{d}+\zeta \cdot \dot{\eta}\right) \mathrm{d} \mathscr{V}$ \\
\hline $\begin{array}{l}\text { Concrete strain energy } \\
\text { (elastic recoverable) }\end{array}$ & $E_{s, c}$ & $\int_{\mathscr{V}}\left(\sigma_{c} \cdot \dot{\varepsilon}_{c}-Y . \dot{d}\right) \mathrm{d} \mathscr{V}$ & $\int_{\mathscr{V}}\left(\sigma_{c} \cdot \dot{\varepsilon}_{c}-\sigma_{c} \cdot \dot{\varepsilon}_{c}^{p}-\sigma^{\pi} \cdot \dot{\varepsilon}^{\pi}-Y \cdot \dot{d}+\zeta \cdot \dot{\eta}\right) \mathrm{d} \mathscr{V}$ \\
\hline $\begin{array}{l}\text { Steel strain energy } \\
\text { (elastic recoverable) }\end{array}$ & $E_{s, s}$ & $\int_{\mathscr{V}}\left(\sigma_{s} \cdot \dot{\varepsilon}_{s}^{e}\right) \mathrm{d} \mathscr{V}$ & $\int_{\mathscr{V}}\left(\sigma_{s} \cdot \dot{\varepsilon}_{s}^{e}\right) \mathrm{d} \mathscr{V}$ \\
\hline Work hardening & $W_{h}$ & $\int_{\mathscr{V}}(Z . \dot{z}) \mathrm{d} \mathscr{V}$ & $\int_{\mathscr{V}}(R \cdot \dot{p}+X . \dot{\alpha}+Z . \dot{z}) \mathrm{d} \mathscr{V}$ \\
\hline Dissipated energy & $E_{d i s}$ & $\int_{\mathscr{V}}(Y . \dot{d}-Z . \dot{z}) \mathrm{d} \mathscr{V}$ & $\int_{\mathscr{V}}\left(\sigma_{c} . \dot{\varepsilon}_{c}^{p}+\sigma^{\pi} . \dot{\varepsilon}^{\pi}+Y . \dot{d}+\zeta . \dot{\eta}-R . \dot{p}-X . \dot{\alpha}-Z . \dot{z}\right) \mathrm{d} \mathscr{Y}$ \\
\hline
\end{tabular}

Table 2: Absorbed energy decomposition

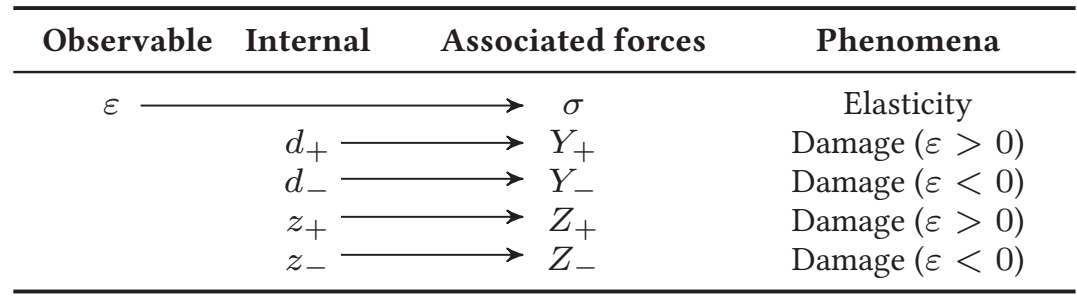

Table 3: BARFRA model variables

\begin{tabular}{cccc}
\hline Observable & Internal & Associated forces & Phenomena \\
\hline$\varepsilon \longrightarrow \sigma^{\pi}$ & Elasticity \\
& $\varepsilon^{\pi} \longrightarrow \sigma$ & Friction \\
$\varepsilon^{p} \longrightarrow$ & Plasticity \\
$\alpha \longrightarrow$ & Friction \\
& & $\longrightarrow$ & Damage \\
$z \longrightarrow$ & Damage \\
$\eta \longrightarrow$ & $\xi$ & Unilateral effect \\
$p \longrightarrow$ & Plasticity \\
\hline
\end{tabular}

Table 4: RICBET model variables

\subsection{Viscous damping identification method}

To study the evolution of viscous damping during nonlinear dynamic computations, an identification method using a single degree-of-freedom model, equivalent to the global structural beam, is developed.

Coupling of modes When the projection on a modal basis is used to solve a dynamic problem, orthogonality of mode is implemented. But, if the damping matrix evolves during computation, 
for example by considering the tangent stiffness matrix, the orthogonality can be altered. So, to evaluate the evolution of modes orthogonality along computation, the matrix $\mathbb{C}^{\text {mod }}$ is computed at different time steps. This matrix is composed by the terms $C_{i j}^{\text {mod }}$ in equation 6.

$$
C_{i j}^{\text {mod }}=\underline{\phi}_{i} \cdot \mathbb{C} \cdot \underline{\phi}_{j} \quad \forall(i, j) \in \llbracket 1 ; N \rrbracket^{2}
$$

where $\left\{\underline{\phi}_{i}\right\}_{i \in \llbracket 1 ; N \rrbracket}$ are the modes determined by a modal analysis on a linear system. So, only matrix $\mathbb{C}$ is evolving during computation in equation 6. By computing $\mathbb{C}^{\text {mod }}$ at different damage levels during dynamic computations, we did not observe significant coupling development. So the resolution of dynamic equation on modal basis remains herein acceptable, even when the tangent stiffness matrix is used.

Modal basis projection Equivalent model of structural element is obtained using a projection of dynamic equation (eq. 3) on modal basis $\mathcal{B}=\left\{\underline{\phi}_{i}\right\}_{i \in \llbracket 1 ; N \rrbracket}$. Displacement vector is defined as a linear combination of modes: $\mathbf{U}=\sum_{i=1}^{N} a_{i} \cdot \underline{\phi}_{i}$ with orthogonal modes: $\underline{\phi}_{i} \cdot \mathbb{M} \cdot \underline{\phi}_{j}=\delta_{i j} \cdot m_{i}$ and $\underline{\phi}_{i} \cdot \mathbb{K} . \underline{\phi}_{j}=\delta_{i j} . k_{i}$ where $\delta_{i j}$ is Kronecker index, and $m_{i}$ and $k_{i}$ are respectively the mass and the stiffness associated to mode $i$. Generally, the basis is mass-normalized, so $m_{i}=1$ and $k_{i}=\omega_{i}^{2}$ where $\omega_{i}=2 \pi f_{i}$ with $f_{i}$ the frequency associated to mode $i$.

The first mode is representing more than $90 \%$ of modal mass, therefore the projection will be done on the first mode only. The displacement is expressed as $\mathbf{U}=a_{1} . \underline{\phi}_{1}$ where $a_{1}$ is time dependent. So, equation 3 in a linear case, and projected on the first mode, becomes equation 7 , and then equation 8 on a normalized format:

$$
\begin{gathered}
\ddot{a}_{1} \cdot \underline{\phi}_{1} \cdot \mathbb{M} \cdot \underline{\phi}_{1}+\dot{a}_{1} \cdot \underline{\phi}_{1} \cdot \mathbb{C} \cdot \underline{\phi}_{1}+a_{1} \cdot \underline{\phi}_{1} \cdot \mathbb{K}_{T} \cdot \underline{\phi}_{1}=-\underline{\phi}_{1} \cdot \mathbb{M} \cdot \underline{\Gamma} \cdot \ddot{\mathbf{U}}_{s} \\
\ddot{a}_{1}+2 \xi \omega_{0} \cdot \dot{a}_{1}+\omega_{D}^{2} \cdot a_{1}=-\frac{\kappa_{1}}{m_{1}} \cdot \ddot{u}_{s}
\end{gathered}
$$

where $\xi$ is the viscous damping ratio, $\omega_{0}$ the initial eigen-frequency, $\omega_{D}$ represents a "damaged" eigen-frequency, $\kappa_{1}=\underline{\phi}_{1} \cdot \mathbb{M} . \underline{\Gamma}$ is the projection coefficient and $\ddot{u}_{s}$ is the seismic acceleration.

Method presentation Demarie \& Sabia [15] proposed a non-linear and non-parametric method to identify non-linear damping and frequency of damaged reinforced concrete elements. The method developed herein is based on this approach. Despite the non-linearity in the studied time responses, we consider that it is possible to decompose the signal in $n_{w}$ windows, such that in each time window the system response can be considered linear. That is to say that, in each window, we are able to evaluate a viscous damping ratio $\xi_{w}$ and a damage eigen-frequency $\omega_{D, w}$ from equation 8 reduced to the window $w$.

After having projected data on the modal basis, a function to identify $f_{i d}$ has to be defined, as well as windows characteristics. On each window, a minimization algorithm is performed to find the best pair of parameters $\left(\xi_{w}, \omega_{D, w}\right)$ using the objective function in equation 9:

$$
\varepsilon_{w}=\frac{\int_{t=0}^{t_{w}}\left(f_{i d, w}-f_{c, w}\right)^{2} \mathrm{~d} t}{\int_{t=0}^{t_{w}}\left(f_{i d, w}\right)^{2} \mathrm{~d} t}
$$

where $f_{i d, w}$ and $f_{c, w}$ are, respectively, the function to identify and the computed function, reduced to the window $w$. Finally, by applying the minimization algorithm in all windows, we obtain the evolution of identified parameters all along the computation. 


\section{STUDIES ON DAMPING MODELS}

\subsection{Dynamic response of the beam}

Comparison of classical damping matrix The first dynamic signal has a very low amplitude. Experimentally, this white-noise signal was performed to characterize the undamaged modal properties of the beam. Numerical responses are well matching experimental ones and we found a first mode eigen-frequency with less than $3 \%$ of error with almost all tested damping formulations. A damping ratio around $0.5 \%$ is required to compute Rayleigh-type matrices.

Then, non-linearities development is studied by applying a high amplitude decreasing sine sweep signal (fig. 3). Damping matrix formulations are compared with experimental data in terms of displacement, acceleration and from constitutive law responses. Viscous damping ratios varying between $0.5 \%$ and $5 \%$ have also been studied. On the contrary to the first linear test, viscous damping ratios around $2 \%$ are most appropriate. This value is smaller than the $5 \%$ often recommended in engineering codes because of the dissipative model used at local scale, the no-yielding of steel reinforcements and the modest strains

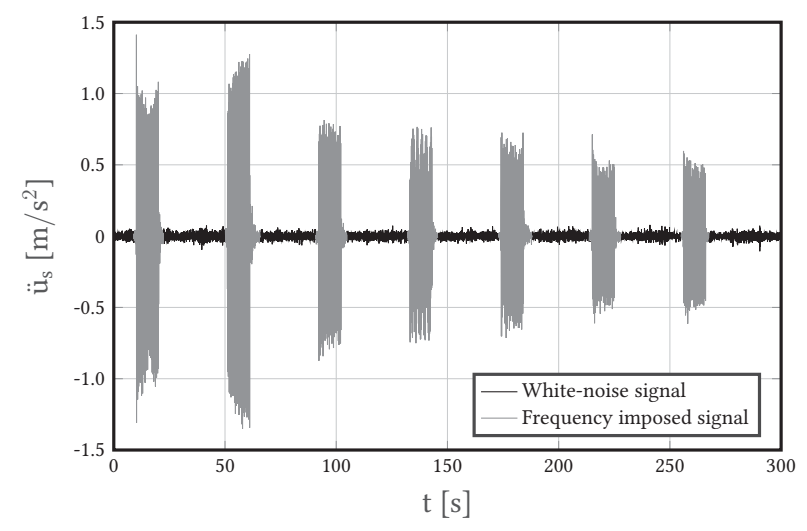

Figure 3: Decreasing sinus sweep - DSS85b and stresses responses. It appears that the viscous damping ratio is strongly dependent on input signal, damping formulations and levels of stress, strain and damage. So, it is impossible to propose a general viscous damping ratio working for all experimental vs numerical simulations. This represents a very significant limitation of viscous damping models computed from classical matrices.

To compare damping matrix formulations, figure 4 synthesizes different results as (i) the error between experimental and numerical data for displacements, accelerations and force responses and (ii) the error in the evolution of eigen-frequencies, and (iii) energy criteria. The best damping formulations are the one given the largest area in spider diagrams. Some formulations are better for some criteria than others. We are still able to say that the most appropriate damping formulations are Rayleigh damping (CRD) and stiffness proportional damping (CKPD) both with the use of tangent stiffness.

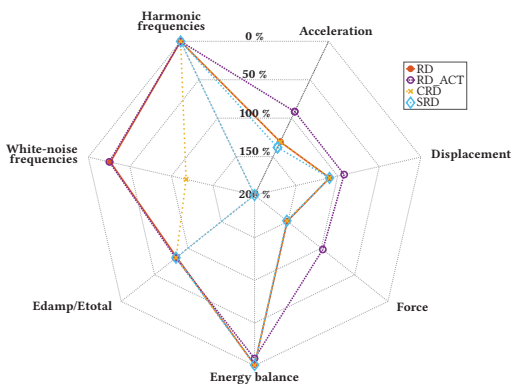

(a) Comparison of RD-type formulations

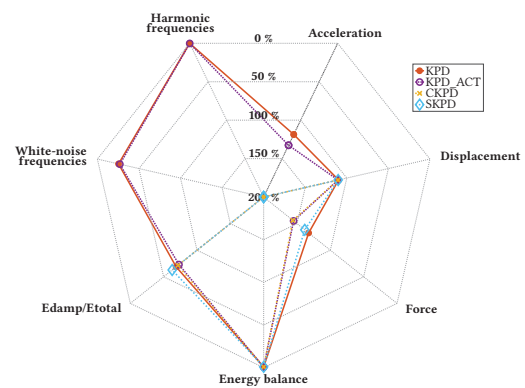

(b) Comparison of KPD-type formulations

Figure 4: General comparison of damping formulations 
Energy dissipations Energies presented in equation 5 are plotted in figure 5a for CKPD damping matrix formulation with a viscous damping ratio of $2 \%$ in case of a damageable (cracking) test (DSS) and with RICBET constitutive model. What is of interest, in this figure, is the distribution of energy between viscous damping and internal local material mechanisms. Indeed, kinematic energy represents a very small part of total energy and is recoverable, so it is null at the end. Generally, viscous damping energy dissipates the most energy. Only stiffness proportional damping matrix leads to a distribution almost $50 \% / 50 \%$. This demonstrates that even with a complex material model, a large amount of energy lacks to be dissipated at material level and has to be dissipated by equivalent viscous damping. Undoubtedly, decreasing the viscous damping ratio leads to a most equal distribution of the two dissipative energy but we saw in dynamic responses that a very small viscous damping ratio does not allow to match experimental data. Finally, to be able to reduce the requirement of viscous damping, we have to dissipate more energy at the material level.

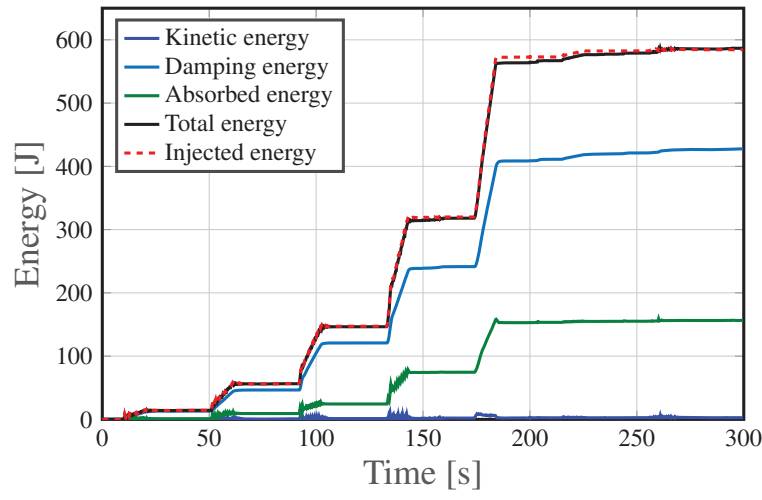

(a) Structural energy balance

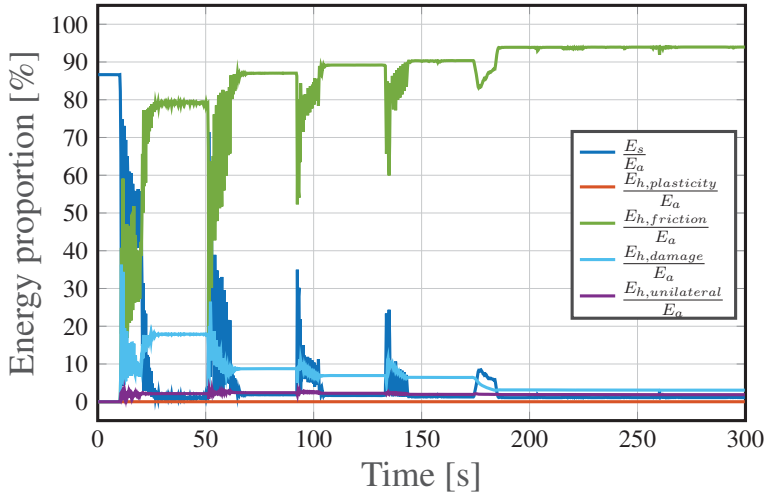

(b) Proportions of dissipative energies at local level

Figure 5: Energy analyses

The RICBET model takes into consideration four dissipative phenomena. Figure $5 \mathrm{~b}$ focuses on hysteretic and strain energies, at local scale. In the beginning, the beam behaves linearly, so no dissipation occurs and strain energy is dominating. Then, cracks open and damage associated energy is dissipated. As soon as cracks open, because they are submitted to an oscillating signal, friction between crack lips develops. We observe that friction fast becomes dominating. When, a mono-harmonic signal segment excites the beams more strongly, cracks pattern evolves leading to an increase in the cracking associated energy proportion (crack propagation) at the expense of friction energy. Finally, plasticity is not dissipating energy because we do not reach concrete compressive non-linearities. Unilateral effect dissipates a small amount of energy during all computation after development of non-linearities.

As a conclusion, we can say that viscous energy is still representing a major part of all dissipative energies demonstrating again the lack of knowledge around the physical representation of local dissipative mechanisms. And, in the case of linear response of steel reinforcements, but nonlinear concrete behavior, the main concrete local dissipative phenomena is the friction, so works should focus on that phenomenon. 


\subsection{Damping coefficient evolution}

Identification method can be applied on experimental or numerical data. Figure 6 particularly focuses on experimental data of two tests. Green data correspond to the lowest amplitude signal (with a maximum acceleration of $1.25 \mathrm{~m} / \mathrm{s}^{2}$ ) while blue data correspond the strongest signal (with a maximum acceleration of $2.25 \mathrm{~m} / \mathrm{s}^{2}$ ). The first test leads to a maximal damage index (modelling the stiffness reduction due to cracking) around 0.6 what we also observed in identification of numerical data. But, in terms of viscous damping ratio, green data are staying around $5 \%$ experimentally while much stronger val-

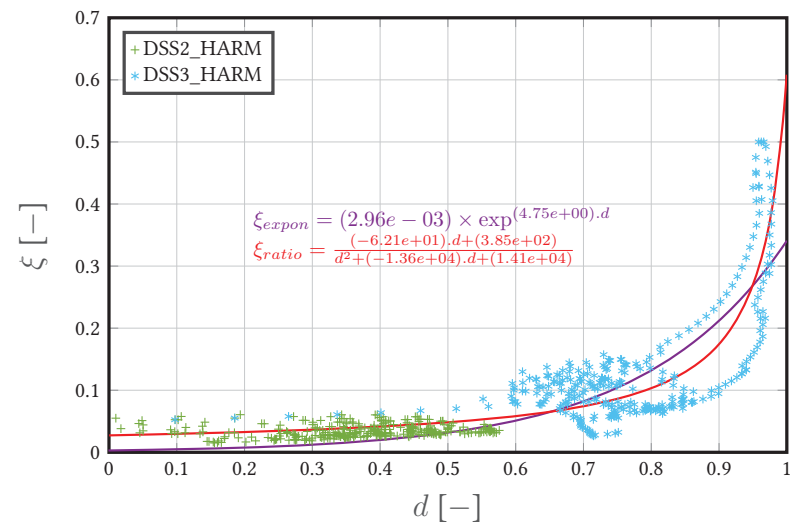

Figure 6: Experimental evolution of identified damping coefficient ues are obtained with numerical identification. This demonstrates that the multi-fiber numerical model is not able to dissipate enough energy at the local scale and that a stronger influence has to be given to viscous damping in numerical computations. This is a huge limitation for the use of viscous damping in non-linear structural analyses.

To conclude on experimental data, the combination of the two more damageable tests shows that the viscous damping ratio can be defined as an exponential (eq. 10) or a polynomial (eq. 11) function of the cracking damage index.

$$
\begin{gathered}
\xi_{\text {expon }}=\left(2.96 \times 10^{-3}\right) \times \exp (4.75 . d) \\
\xi_{\text {ratio }}=\frac{-62.1 . d+385}{d^{2}-1.36 \times 10^{4} . d+1.41 \times 10^{4}}
\end{gathered}
$$




\section{CONCLUSIONS}

The modeling of damping in structural non-linear dynamic computations was discussed in this paper using different analyses. First, an exhaustive numerical campaign was performed to characterize dissipations in reinforced concrete elements submitted to earthquake excitations and tested on a shake table. Particularly, sixteen classical damping matrix formulations proposed in literature have been compared. Results demonstrates that it is difficult to determine one type of damping working for all signal intensities. The damping formulation have to be selected depending on input signal intensity. However, using tangent stiffness matrix in Rayleigh damping formulation with or without the mass proportional part appears the best choice herein to represent the dynamic experimental responses. Rayleigh damping matrix evaluation requires to impose damping ratios for some modes frequencies. Analyses performed on multi-fiber model as well as on identification studies with a $1 \mathrm{D}$ model show that a constant value of damping ratio all along computation does not give accurate results when non-linearities occur. Exponential evolutions of damping coefficient as a function of internal damage characterized by stiffness degradation due to concrete cracking seems to better model physical phenomena and so to improve correlation with experimental data.

The works to follow will propose a new formulation of damping matrix based on local variables. The aim is to increase physical basis of the damping matrix. Preliminary results are showing good accuracy in comparisons with experimental data. But some developments still remain to compare local variables associated to different phenomena, like friction, and determine predictive functions to compute damping matrix.

\section{ACKNOWLEDGMENTS}

- The authors wish to thank SEISM Institute for financing a part of the research and development performed at École Polytechnique of Montréal.

- This work was performed using HPC resources from the "Mésocentre" computing center of CentraleSuplec and École Normale Supérieure Paris-Saclay supported by CNRS and Région Île-de-France (http://mesocentre.centralecentralesupelec.fr). 


\section{REFERENCES}

[1] J. F. Hall, Problems encountered from the use (or misuse) of Rayleigh damping. Earthquake Engineering and Structural Dynamics, 35:525-545, 2005.

[2] T. Heitz, A. Le Maoult, B. Richard, C. Giry, F. Ragueneau, Dissipations in reinforced concrete components: Static and dynamic experimental identification strategy. Engineering Structures, 163:436-451, 2018.

[3] J. W. S. B. Rayleigh, The theory of sound - Volume II. Macmillan, 1896.

[4] E. Spacone, F. C. Filippou, F. F. Taucer, Fiber beam-column model for non-linear analysis of R/C frames: Part I. Formulation. Earthquake Engineering \& Structural Dynamics, 25(7):711-725, 1996.

[5] S. Moulin, Code_Aster : Élément de poutre multifibre (droite). Manuel de Référence R3.08.08, 2010.

[6] S. Capdevielle, Comportement des grands ouvrages de Génie Civil - Modélisation par éléments poutres multifibres. PhD thesis, 2017.

[7] R. Crambuer, Contribution à l'identification de l'amortissement : Approches expérimentales et numériques. PhD thesis, 2013.

[8] B. Richard, F. Ragueneau, Continuum damage mechanics based model for quasi brittle materials subjected to cyclic loadings: Formulation, numerical implementation and applications. Engineering Fracture Mechanics, 98:383-406, 2013.

[9] M. J. N. Priestley, D. N. Grant, Viscous damping in seismic design and analysis. Journal of Earthquake Engineering, 09(2):229-255, 2005.

[10] L. Petrini, C. Maggi, M J. N. Priestley, G. M. Calvi, Experimental verification of viscous damping modelling for inelastic time history analyzes. Journal of Earthquake Engineering, 12(1):125-145, 2008.

[11] E. L. Wilson, J. Penzien, Evaluation of orthogonal damping matrices. International Journal for Numerical Methods in Engineering, 4(1):5-10, 1972.

[12] A. Chopra, F. McKenna, Modelling viscous damping in nonlinear response history analysis of buildings for earthquake excitations: Modal damping. Earthquake Engineering \& Structural Dynamics, 45(2):193-211, 2016.

[13] C.-M. Uang, V. V. Bertero, Evaluation of seismic energy in structures. Earthquake Engineering \& Structural Dynamics, 19(1):77-90, 1990.

[14] K. Low, A modified Dunkerley formula for eigenfrequencies of beams carrying concentrated masses. International Journal of Mechanical Sciences, 42(7):1287-1305, 2000.

[15] G. V. Demarie, D. Sabia, Non-linear damping and frequency identification in a progressively damaged R.C. element. Experimental Mechanics, 51(2):229-245, 2011.

[16] T. Heitz, C. Giry, B. Richard, F. Ragueneau, Identification of an equivalent viscous damping function depending on engineering demand parameters. Engineering Structures, 188:637-649, 2019. 